PAPER

\title{
Model Reduction with Time Delay Combining Least-Squares Method with Artificial Bee Colony Algorithm
}

\author{
Tomohiro Hachino $^{1}$, Kosuke Sameshima ${ }^{1}$, Hitoshi Takata ${ }^{1}$, Shigeru Nakayama ${ }^{1}$, \\ Ichiro Iimura $^{2}$, Seiji Fukushima ${ }^{1}$ and Yasutaka Igarashi ${ }^{1}$ \\ ${ }^{1}$ Kagoshima University, 1-21-40 Korimoto, Kagoshima 890-0065, Japan \\ 2 Prefectural University of Kumamoto, 3-1-100 Tsukide, Higashi-ku, Kumamoto 862-8502, Japan \\ E-mail: hachino@eee.kagoshima-u.ac.jp
}

\begin{abstract}
In this paper we propose a novel method of model reduction with a time delay for single-input, single-output continuous-time systems by a separable least-squares (LS) approach. The reduced-order model is determined by minimizing the integral of the magnitude squared of the transfer function error. The denominator parameters and time delay of the reduced-order model are represented by the positions of the food sources of the employed bees and searched for by the artificial bee colony algorithm, while the numerator parameters are estimated by the linear LS method for each candidate of the denominator parameters and time delay. All the best parameters and the time delay of the reduced-order model are obtained through the search by the employed, onlooker and scout bees. Simulation results show that the accuracy of the proposed method is superior to that of the genetic algorithm (GA)-based model reduction algorithm.
\end{abstract}

Keywords: model reduction, time delay, separable least-squares, artificial bee colony algorithm

\section{Introduction}

The problem of approximating high-order systems by low-order models is one of the important problems in system theory. The use of a reduced-order model makes it more efficient to implement system analyses, predictions and control designs. Model reduction problems have been discussed using a number of approaches [1-11]. One of the popular approaches is the $L_{2}$ optimal model reduction. In this approach, the reduced-order model is generally determined so that a quadratic cost, i.e., the integral of the magnitude squared of the transfer function error between the original system and the reduced-order model, is minimized.

Many real systems have inherently pure time delays, and systems of multiple first-order lags in a cascade also perform as if they are time delay systems [7]. When such systems are approximated by only rational transfer functions, the order of the reduced model has to be high for good approximation. If an additional time delay is introduced into the reduced-order model, the approximation might be greatly improved with fewer parameters of the rational part. Although some model reduction methods with a time delay have been presented [1,5-10], the time delay is known a priori [5], searched for one by one [6] or calculated by a gradient-based method $[7,9]$.

The minimization of the above-mentioned quadratic cost function is a nonlinear problem with respect to the denominator parameters and the time delay of the reduced-order model. Therefore, we have to solve a very complicated nonlinear optimization problem for $L_{2}$ model reduction. For this problem, one of the authors proposed a hybrid model reduction algorithm combining the linear least-squares (LS) method with the genetic algorithm (GA) [10,11]. However the GA has many setting parameters and requires a complicated coding technique and genetic operations. Moreover, the GA has high potential for global optimization but it is slightly inferior in local search ability.

To improve the accuracy and efficiency of model reduction, in this paper, we propose a novel $L_{2}$ model reduction algorithm for single-input, single-output continuous-time systems. The proposed model reduction algorithm is based on the separable LS approach combining the linear LS method with the artificial bee 
colony (ABC) algorithm. The concept of the separable LS approach was originally proposed in [12] and has been utilized for linear and nonlinear system identification [13-15]. The ABC algorithm is an optimization algorithm inspired by the intelligent behavior of honeybee swarms [16]. This algorithm consists of searching by three types of bees: employed bees, onlooker bees and scout bees. Similarly to the GA, the ABC algorithm searches from not a single point but a population of points and only uses information about the objective function, not derivatives or other auxiliary knowledge. The advantages of the ABC algorithm are summarized as follows [16-18]:

(i) The ABC algorithm consists of only the basic arithmetic operations and does not require complicated coding and genetic operations such as crossovers and mutations.

(ii) There are very few setting parameters in the ABC algorithm.

(iii) The performance of the ABC algorithm is better than or similar to those of other population-based algorithms.

(iv) The ABC algorithm is robust to increases in the dimension of the search space.

These advantages suggest that the use of the ABC algorithm will improve the accuracy and efficiency of model reduction, especially in the case that the order of the reduced model is high.

Note that if the denominator parameters and time delay of the reduced-order model are fixed a priori, the determination of the numerator parameters becomes a linear problem, and they are easily obtained by the linear LS method. Therefore, we propose a separable LS approach combining the linear LS method with the ABC algorithm to determine reduced-order models. The denominator parameters and time delay of the reduced-order model are represented by the positions of the food sources of the employed bees and searched for by the ABC algorithm, while the numerator parameters are estimated by the linear LS method for each candidate of the denominator parameters and time delay. The fitness values are evaluated as the inverse of the integral of the magnitude squared of the transfer function errors between the original system and the candidates of the reduced-order model. All the best parameters and the time delay of the reduced-order model are obtained through searching by the employed, onlooker and scout bees.

This paper is organized as follows. In Sect. 2 the problem is formulated. In Sect. 3 the numerator parameters of the reduced-order model are represented as a function of the denominator parameters and time delay. Then the model reduction algorithm is given by a separable LS approach that combines the linear LS method with the ABC algorithm. In Sect. 4 simulation results are shown to illustrate the effectiveness of the proposed method. Finally, some conclusions are given in Sect. 5 .

\section{Statement of the Problem}

Consider an $n$ th-order single-input, single-output time delay system with the transfer function

$$
\begin{aligned}
& G(s) \\
& =\frac{\beta_{1} s^{m-1}+\beta_{2} s^{m-2}+\cdots \cdots+\beta_{m}}{s^{n}+\alpha_{1} s^{n-1}+\alpha_{2} s^{n-2}+\cdots \cdots+\alpha_{n}} \exp (-\tau s)
\end{aligned}
$$

where the rational part is stable and strictly proper.

Let the $\ell$ th-order reduced model with the time delay be given by

$$
\begin{aligned}
& \widetilde{G}(s) \\
& =\left\{\begin{array}{c}
\frac{d_{1} s^{k-1}+d_{2} s^{k-2}+\cdots \cdots+d_{k}}{\prod_{i=1}^{[\ell / 2]}\left(s^{2}+a_{i} s+b_{i}\right)} \exp (-T s) \\
\frac{d_{1} s^{k-1}+d_{2} s^{k-2}+\cdots \cdots+d_{k}}{\left\{\prod_{i=1}^{[\ell / 2]}\left(s^{2}+a_{i} s+b_{i}\right)\right\}(s+c)} \exp (-T s) \\
(\ell \text { : odd })
\end{array}\right.
\end{aligned}
$$

where $\ell<n$ and [.] denotes Gauss's symbol. The rational parts of Eq. (2) are also strictly proper. Note that the form of the denominator in Eq. (2) enables us to express all possible patterns of the poles and guarantees the stability of the reduced-order model when all denominator parameters $\left\{a_{i}\right\},\left\{b_{i}\right\}$ and $c$ are positive.

Our goal is to determine the parameters $\left\{a_{i}\right\},\left\{b_{i}\right\}$, $c,\left\{d_{i}\right\}$ and the time delay $T$ of the stable reducedorder model so that the integral of the magnitude squared of the weighted transfer function error

$$
J=\int_{-\infty}^{+\infty}|\{G(j \omega)-\widetilde{G}(j \omega)\} W(j \omega)|^{2} d \omega
$$

is minimized. Here $W(j \omega)$ is a frequency-weighting function, which is introduced to obtain a better approximation over a prespecified frequency range such as near resonances and operating frequencies.

\section{Model Reduction Algorithm}

\subsection{Obtaining numerator parameters by LS method}

In this section, we show that the numerator parameters of the reduced-order model are represented as a function of the denominator parameters and time delay. In the following, only the odd case of Eq. (2) is considered, because the even case of Eq. (2) can be treated in the same manner.

The reduced-order model (Eq. (2)) can be rewritten as 


$$
\begin{aligned}
& \widetilde{G}(j \omega)=\boldsymbol{z}^{T}(j \omega, \boldsymbol{X}) \boldsymbol{\theta} \\
& \boldsymbol{z}^{T}(j \omega, \boldsymbol{X}) \\
& =\frac{\left[\begin{array}{lllll}
(j \omega)^{k-1} & (j \omega)^{k-2} & \cdots & j \omega & 1
\end{array}\right]}{[\ell / 2]} \\
& \prod_{i=1}\left\{(j \omega)^{2}+a_{i}(j \omega)+b_{i}\right\}\{(j \omega)+c\} \\
& \times \exp (-j \omega T)
\end{aligned}
$$

where

$$
\begin{aligned}
& \boldsymbol{X}^{T}=\left[\begin{array}{lllllll}
a_{1} & b_{1} & \cdots & a_{[\ell / 2]} & b_{[\ell / 2]} & c & T
\end{array}\right] \\
& \boldsymbol{\theta}^{T}=\left[\begin{array}{lllll}
d_{1} & d_{2} & \cdots & d_{k-1} & d_{k}
\end{array}\right]
\end{aligned}
$$

$\boldsymbol{X}$ consists of the denominator parameters and time delay, and $\boldsymbol{\theta}$ consists of the numerator parameters of the reduced-order model. Then the cost function (Eq. (3)) is given using Eq. (4) as follows:

$$
\begin{aligned}
& J(\boldsymbol{X}, \boldsymbol{\theta}) \\
& =\int_{-\infty}^{+\infty}\left|\left\{G(j \omega)-\boldsymbol{z}^{T}(j \omega, \boldsymbol{X}) \boldsymbol{\theta}\right\} W(j \omega)\right|^{2} d \omega \\
& =\int_{-\infty}^{+\infty}\left([W(j \omega) G(j \omega)]_{\Re}-\left[W(j \omega) \boldsymbol{z}^{T}(j \omega, \boldsymbol{X})\right]_{\Re} \boldsymbol{\theta}\right)^{2} \\
& \quad+\left([W(j \omega) G(j \omega)]_{\Im}-\left[W(j \omega) \boldsymbol{z}^{T}(j \omega, \boldsymbol{X})\right]_{\Im} \boldsymbol{\theta}\right)^{2} d \omega
\end{aligned}
$$

where $[\cdot]_{\Re}$ and $[\cdot]_{\Im}$ denote the real and imaginary parts of the complex number, respectively. Thus, applying the linear LS method to Eq. (6), the numerator parameter vector $\boldsymbol{\theta}$ is obtained as a function of $\boldsymbol{X}$ :

$$
\begin{aligned}
& \boldsymbol{\theta}(\boldsymbol{X}) \\
& =\left[\int_{-\infty}^{+\infty}[W(j \omega) \boldsymbol{z}(j \omega, \boldsymbol{X})]_{\Re}\left[W(j \omega) \boldsymbol{z}^{T}(j \omega, \boldsymbol{X})\right]_{\Re}\right. \\
& \left.\quad+[W(j \omega) \boldsymbol{z}(j \omega, \boldsymbol{X})]_{\Im}\left[W(j \omega) \boldsymbol{z}^{T}(j \omega, \boldsymbol{X})\right]_{\Im} d \omega\right] \\
& \times\left[\int_{-\infty}^{+\infty}[W(j \omega) \boldsymbol{z}(j \omega, \boldsymbol{X})]_{\Re}[W(j \omega) G(j \omega)]_{\Re}\right. \\
& \left.\quad+[W(j \omega) \boldsymbol{z}(j \omega, \boldsymbol{X})]_{\Im}[W(j \omega) G(j \omega)]_{\Im} d \omega\right]
\end{aligned}
$$

\subsection{Outline of $\mathrm{ABC}$ algorithm}

The ABC algorithm is an optimization algorithm inspired by the behavior of real honeybees [16]. In this algorithm, a colony of artificial bees consists of three groups of bees: employed bees, onlooker bees and scout bees. The roles of these groups are as follows:

(1) Employed bees

The employed bees determine a food source within the neighborhood of a food source in their memory.
The size of employed bees is half of the colony size. Every employed bee works on only one food source. Therefore, the number of employed bees is equal to the number of food sources. The employed bees evaluate the profitability of food sources such as the amount of nectar, and share their information with the onlooker bees in the hive. A employed bee that has worked on an abandoned food source is differentiated into a scout bee.

(2) Onlooker bees

The onlooker bees waiting in the hive select one food source through the information obtained from the employed bees' dances and search in the neighborhood of the selected food source. This selection is implemented by "roulette-wheel" slots weighted in proportion to the profitability of the food source. Therefore, the onlooker bees are likely to search around more profitable food sources. The size of onlooker bees is also half of the colony size.

(3) Scout bees

The scout bees differentiated from the employed bees search for new food sources randomly.

In the optimization problem, the positions of the food sources correspond to the candidates of the solution and the profitability of the food source corresponds to the fitness value, which represents the goodness of the solution. A suboptimal solution is obtained by repeatedly performing searches using the employed, onlooker and scout bees.

\subsection{Model reduction by $\mathrm{ABC}$ algorithm}

As the cost function $J$ of Eq. (3) or Eq. (6) generally has multiple local minima, this reduction becomes a nonlinear optimization problem. However, we can separate the linear optimization part and the nonlinear optimization part for this optimization problem. Note that if the candidates of the vector $\boldsymbol{X}$, which consists of the denominator parameters and time delay, are given, the numerator parameter vector $\boldsymbol{\theta}$ can be estimated by the linear LS method as shown in Eq. (7). Therefore, in this paper we present the model reduction algorithm by a separable LS approach combining the linear LS method with the ABC algorithm. The candidates of $\boldsymbol{X}$ are represented by the positions of the food sources and searched for by the ABC algorithm, where the candidates of the numerator parameter vector $\boldsymbol{\theta}$ are estimated by the linear LS method.

Of course, it is possible to optimize both $\boldsymbol{X}$ and $\boldsymbol{\theta}$ directly using only the $\mathrm{ABC}$ algorithm. However, such a naive method makes the $\mathrm{ABC}$ algorithm computationally demanding because the dimension of the search space increases. The proposed separable LS approach is more efficient in that it reduces the dimension of the search space by about half.

The proposed model reduction algorithm is described as follows: 


\section{Step 1: Initialization}

(1-1) Generate an initial population of $N_{s}$ bees with random positions of the food sources $\boldsymbol{X}_{i}(i=$ $\left.1,2, \cdots, N_{s}\right)$ from Eq. (8):

$$
\begin{gathered}
X_{i j}=X_{m i n, j}+\operatorname{rand}[0,1] \cdot\left(X_{\max , j}-X_{m i n, j}\right) \\
(j=1,2, \cdots, \ell+1)
\end{gathered}
$$

where $N_{s}$ denotes the size of employed bees or onlooker bees and $X_{i j}$ is the $j$ th element of the vector $\boldsymbol{X}_{i} . \quad X_{\min , j}$ and $X_{\max , j}$ are the minimum and maximum values of $X_{i j}$, respectively. $\operatorname{rand}[0,1]$ is a uniformly distributed random number with amplitude in the range $[0,1]$.

(1-2) Set the iteration counter $l$ to 1 .

(1-3) Set the counter for abandonment trial $l_{i}$ to 0 . The counter trial $_{i}$ is the number of times that the solution $\boldsymbol{X}_{i}$ is not improved by the employed and onlooker bees.

\section{Step 2: Estimation of numerator parameter $\theta$}

Calculate the numerator parameter vector $\boldsymbol{\theta}_{i}$ from

Eq. (7) by the linear LS method for $\boldsymbol{X}_{i}(i=1,2, \cdots$, $N_{s}$ ). In practice, the integral in Eq. (7) is carried out by a numerical integration method such as the trapezoidal rule with a reasonable range $\left[0, \omega_{f}\right]$.

Step 3: Fitness value calculation

Calculate the fitness values from Eq. (9):

$$
\begin{aligned}
& F_{i}\left(\boldsymbol{X}_{i}, \boldsymbol{\theta}_{i}\right) \\
& =\frac{1}{\int_{0}^{\omega_{f}}\left|\left\{G(j \omega)-\boldsymbol{z}^{T}\left(j \omega, \boldsymbol{X}_{i}\right) \boldsymbol{\theta}_{i}\right\} W(j \omega)\right|^{2} d \omega}
\end{aligned}
$$

where the range of the integral is set to $\left[0, \omega_{f}\right]$ as in step 2. Note that the fitness value function is the inverse of the cost function (Eq. (6)), i.e., maximizing the fitness value function means minimizing the cost function.

\section{Step 4: Search by the employed bees}

(4-1) Determine the new positions of the food sources $\boldsymbol{V}_{i}$ around $\boldsymbol{X}_{i}$ for the employed bees from Eq. (10):

$$
\begin{array}{r}
V_{i j}=X_{i j}+\operatorname{rand}[-1,1] \cdot\left(X_{i j}-X_{k j}\right) \\
(j=1,2, \cdots, \ell+1)
\end{array}
$$

where $V_{i j}$ is the $j$ th element of the vector $\boldsymbol{V}_{i}$ and $k$ is a random integer selected from $\left\{1,2, \cdots, N_{s}\right\}$, where $k \neq i$.

(4-2) Calculate the numerator parameter vector $\boldsymbol{\vartheta}_{i}$ from Eq. (7) by the linear LS method for $\boldsymbol{V}_{i}(i=$ $\left.1,2, \cdots, N_{s}\right)$.

(4-3) Calculate the fitness values from Eq. (11):

$$
=\frac{F_{i}\left(\boldsymbol{V}_{i}, \boldsymbol{\vartheta}_{i}\right)}{\int_{0}^{\omega_{f}}\left|\left\{G(j \omega)-\boldsymbol{z}^{T}\left(j \omega, \boldsymbol{V}_{i}\right) \boldsymbol{\vartheta}_{i}\right\} W(j \omega)\right|^{2} d \omega}
$$

(4-4) If $F_{i}\left(\boldsymbol{X}_{i}, \boldsymbol{\theta}_{i}\right)<F_{i}\left(\boldsymbol{V}_{i}, \boldsymbol{\vartheta}_{i}\right)$, update $\boldsymbol{X}_{i}, \boldsymbol{\theta}_{i}$ and $F_{i}\left(\boldsymbol{X}_{i}, \boldsymbol{\theta}_{i}\right)$ with $\boldsymbol{V}_{i}, \boldsymbol{\vartheta}_{i}$ and $F_{i}\left(\boldsymbol{V}_{i}, \boldsymbol{\vartheta}_{i}\right)$, respectively, and set trial $_{i}=0$. Otherwise set trial $_{i}=$ trial $_{i}+1$. This procedure is called "greedy selection".

\section{Step 5: Search by the onlooker bees}

(5-1) Choose one position of the food source for each onlooker bee from $\boldsymbol{X}_{i}\left(i=1,2, \cdots, N_{s}\right)$ through "roulette-wheel" slots weighted in proportion to the fitness value of the employed bee. Namely, each onlooker bee selects one position of the food source with probability $F_{i}\left(\boldsymbol{X}_{i}, \boldsymbol{\theta}_{i}\right) / \sum_{p=1}^{N_{s}} F_{p}\left(\boldsymbol{X}_{p}, \boldsymbol{\theta}_{p}\right)$.

(5-2) Calculate the new positions of the food sources $\boldsymbol{V}_{i}$ corresponding to the selected positions $\boldsymbol{X}_{i}$ from Eq. $(10)$.

(5-3) Calculate the numerator parameter vector $\boldsymbol{\vartheta}_{i}$ from Eq. (7) for $\boldsymbol{V}_{i}$.

(5-4) Calculate the fitness values $F_{i}\left(\boldsymbol{V}_{i}, \boldsymbol{\vartheta}_{i}\right)$ from Eq. (11).

(5-5) Carry out the greedy selection in the same way as Step 4 (4-4).

\section{Step 6: Search by the scout bees}

If the counter for abandonment trial ${ }_{i}$ is greater than or equal to the prespecified number limit, carry out the following procedure.

(6-1) Differentiate the corresponding employed bee into the scout bee and generate the new position of the food source $\boldsymbol{X}_{i}$ for the scout bee randomly from Eq. (8).

(6-2) Calculate the numerator parameter vector $\boldsymbol{\theta}_{i}$ for $\boldsymbol{X}_{i}$ from Eq. (7).

(6-3) Calculate the fitness values $F_{i}\left(\boldsymbol{X}_{i}, \boldsymbol{\theta}_{i}\right)$ from Eq. (9).

This step means that if the solution is not improved limit times through the search by the employed and onlooker bees, the corresponding employed bee gives up the search around its food source and transforms itself into a scout bee to search around a randomly selected food source. Since the number limit is usually set to be the product of the employed bee size and the dimension of the search space [16], this number is taken to be limit $=N_{s}(\ell+1)$ in this paper.

\section{Step 7: Repetition}

Set the iteration counter to $l=l+1$ and go to Step 4 until the prespecified iteration number $l_{\max }$.

Finally at the termination of this algorithm when $l=l_{\max }$, the suboptimal $\hat{\boldsymbol{X}}$ and the corresponding $\hat{\boldsymbol{\theta}}$ are determined by the best position of the food source.

\section{Numerical Simulations}

Consider the fifth-order nonminimal phase system with time delay described by the following transfer function $[9]$ :

$$
G(s)=\frac{(s+1)(s-1)(s+10)}{(s+2)^{3}(s+3)(s+4)} \exp (-0.5 s)
$$



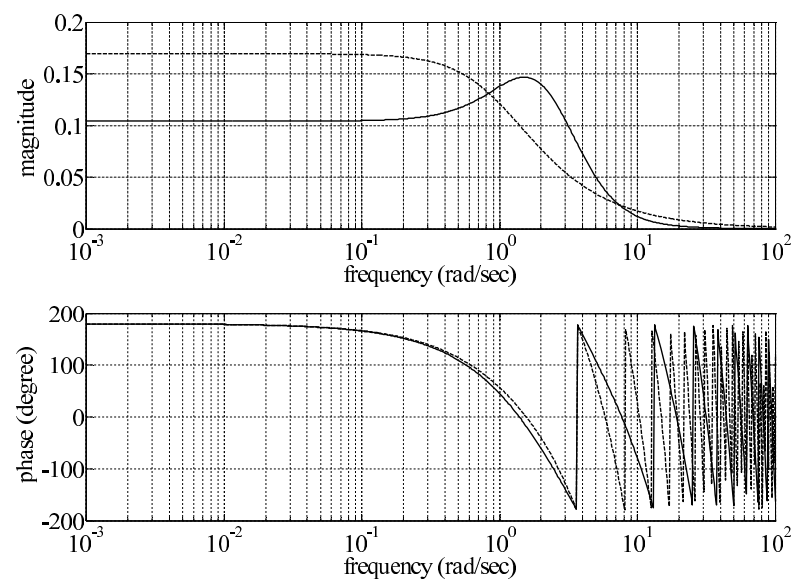

Fig. 1 Bode plots of the original system and reducedorder model $\left(-G(s),---\widetilde{G}_{1}(s)\right)$
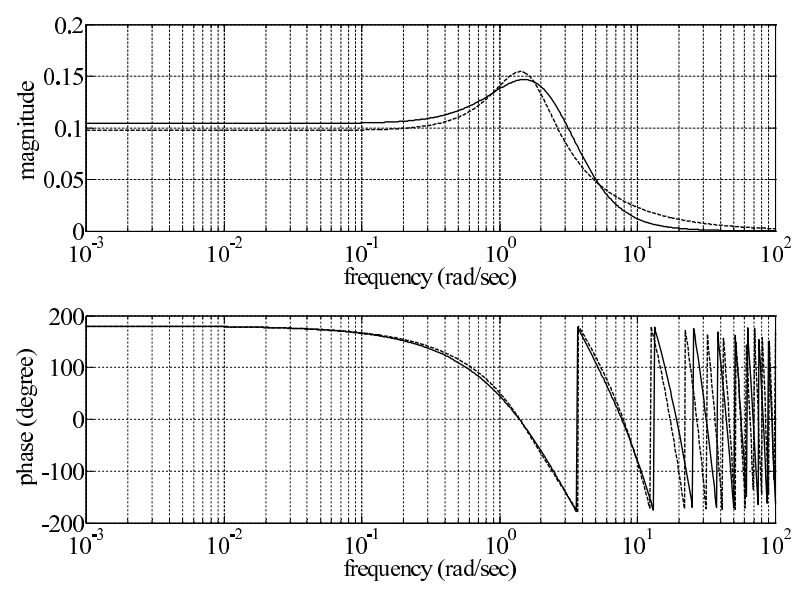

Fig. 2 Bode plots of the original system and reducedorder model $\left(-G(s),---\widetilde{G}_{2}(s)\right)$

The setting parameters of the ABC algorithm are chosen as follows:

(i) employed bee size $N_{s}=\ell \times 100$

(50\% of the colony size)

(ii) maximum iteration number $l_{\max }=100$

The first-order, second-order, third-order and fourth-order reduced models with the time delay obtained by the proposed algorithm are

$$
\begin{aligned}
& \widetilde{G}_{1}(s)=\frac{-0.17274}{s+1.0196} \exp (-1.3770 s) \\
& \widetilde{G}_{2}(s)=\frac{0.22639 s-0.24948}{s^{2}+1.8157 s+2.5618} \exp (-0.64562 s) \\
& \widetilde{G}_{3}(s)=\frac{-0.00032451 s^{2}+1.3507 s-1.3486}{\left(s^{2}+3.5896 s+6.0218\right)(s+2.0623)} \\
& \quad \times \exp (-0.51710 s)
\end{aligned}
$$
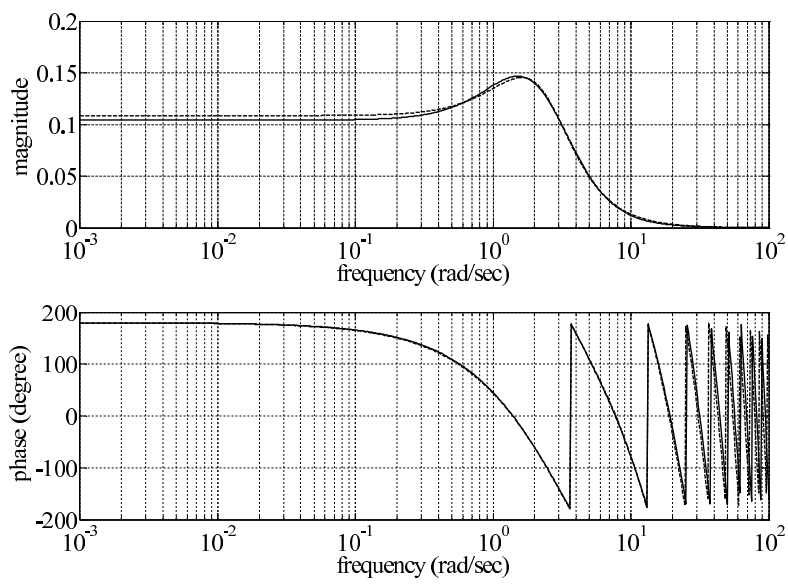

Fig. 3 Bode plots of the original system and reducedorder model $\left(-G(s),---\widetilde{G}_{3}(s)\right)$
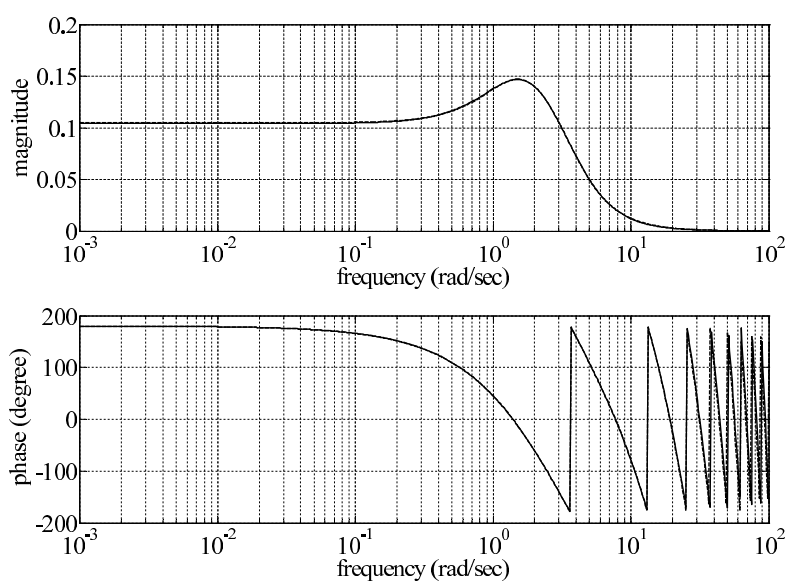

Fig. 4 Bode plots of the original system and reducedorder model $\left(-G(s),---\widetilde{G}_{4}(s)\right)$

$$
\begin{aligned}
& \widetilde{G}_{4}(s) \\
& =\frac{-0.0024248 s^{3}+1.1961 s^{2}+2.5516 s-3.7625}{\left(s^{2}+5.0581 s+12.474\right)\left(s^{2}+2.6364 s+2.8755\right)} \\
& \quad \times \exp (-0.51012 s)
\end{aligned}
$$

where the frequency-weighting function $W(s)=1$ (unity weighting). Note that in practice the use of the first-order reduced model is not realistic if the model reduction is performed for a control design, because the high-order system (Eq. (12)) has resonance characteristics.

The Bode plots of the original system $G(s)$ and the reduced-order models $\widetilde{G}_{1}(s), \widetilde{G}_{2}(s), \widetilde{G}_{3}(s)$ and $\widetilde{G}_{4}(s)$ are shown in Figs. $1-4$, and their impulse responses are shown in Figs. 5-8, respectively. The step responses of $\widetilde{G}_{1}(s)$ and $\widetilde{G}_{2}(s)$ are also respectively shown in Figs. 9 and 10, which are compared with those of the frequency-weighted reduced-order 


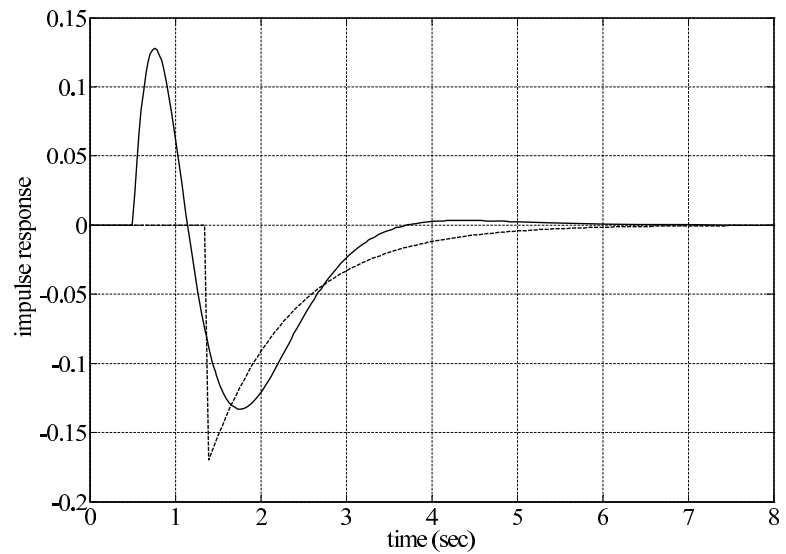

Fig. 5 Impulse responses of the original system and reduced-order model $\left(-G(s),---\widetilde{G}_{1}(s)\right)$

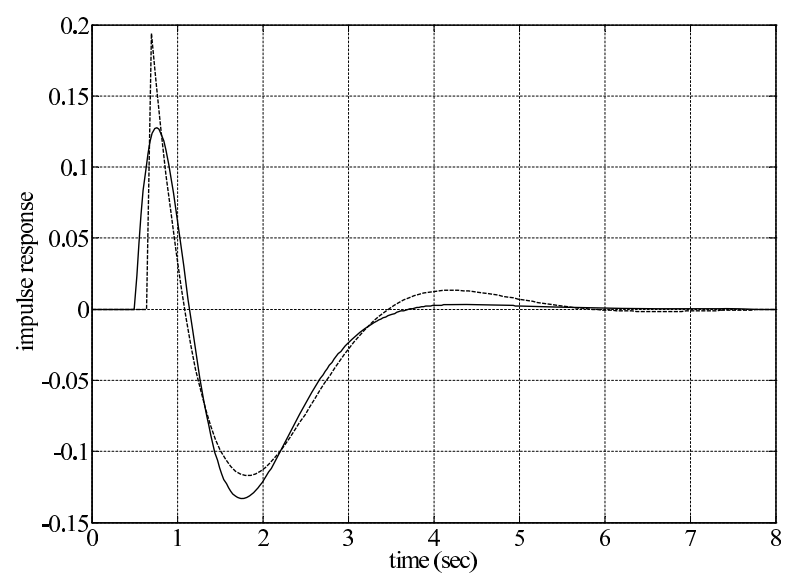

Fig. 6 Impulse responses of the original system and reduced-order model $\left(-G(s),---\widetilde{G}_{2}(s)\right)$

models later. Although the third-order and fourthorder reduced models match the original system well, the first-order and second-order reduced models have quite large approximation errors at low frequencies. Since many practical systems operate in low-frequency ranges, we proceed to the frequency-weighted model reduction to obtain a better approximation at low frequencies. The following frequency-weighting function with low-pass characteristics

$$
W(s)= \begin{cases}\frac{1}{(s+0.1)^{2}} & (\ell=1) \\ \frac{1}{(s+3)^{2}} & (\ell=2)\end{cases}
$$

is introduced. The first-order and second-order frequency-weighted reduced models are obtained as follows:

$$
\widetilde{G}_{1 w}(s)=\frac{-0.060204}{s+0.57001} \exp (-0.37928 s)
$$

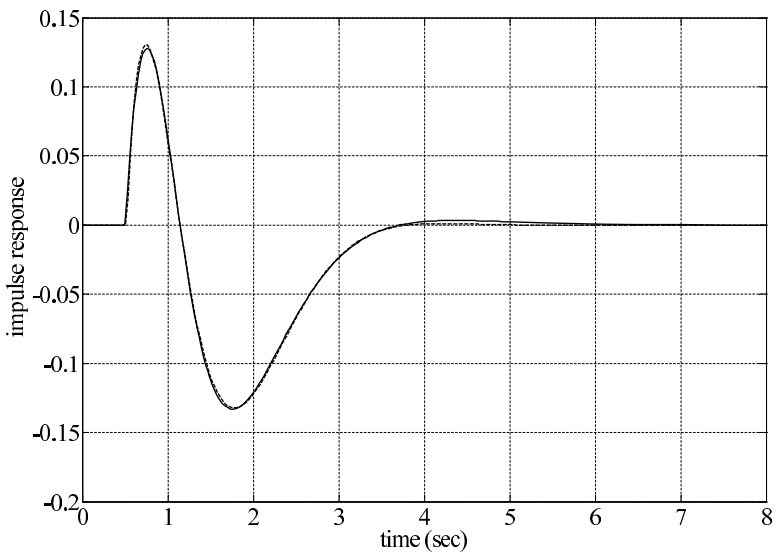

Fig. 7 Impulse responses of the original system and reduced-order model $\left(-G(s),--\widetilde{G}_{3}(s)\right)$

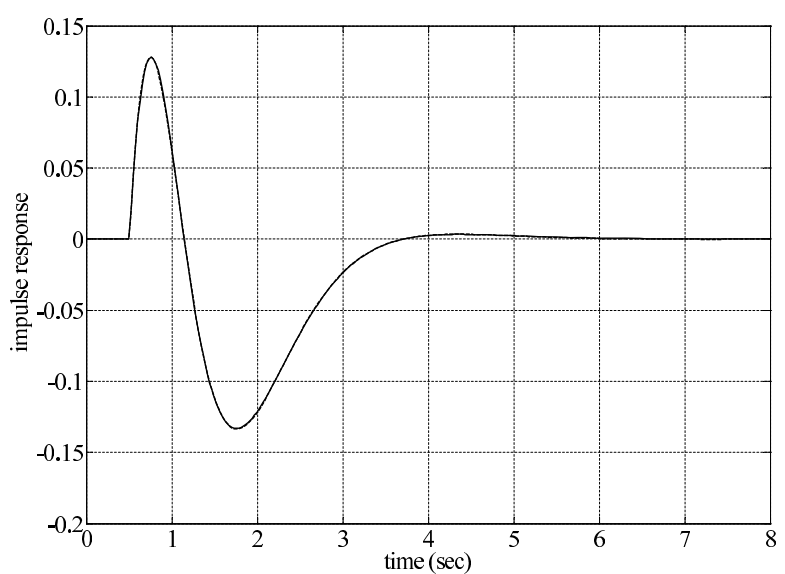

Fig. 8 Impulse responses of the original system and reduced-order model $\left(-G(s),---\widetilde{G}_{4}(s)\right)$

$$
\widetilde{G}_{2 w}(s)=\frac{0.30524 s-0.31830}{s^{2}+2.4606 s+3.0415} \exp (-0.69454 s)
$$

The Bode plots of the original system $G(s)$ and the frequency-weighted reduced-order models $\widetilde{G}_{1 w}(s)$ and $\widetilde{G}_{2 w}(s)$ are shown in Figs. 11 and 12, and their impulse responses are shown in Figs. 13 and 14 , respectively. The step responses of $\widetilde{G}_{1 w}(s)$ and $\widetilde{G}_{2 w}(s)$ are also shown in Figs. 15 and 16, respectively. Although it is difficult to judge which is better, the impulse response of $\widetilde{G}_{1}(s)$ or the impulse response of $\widetilde{G}_{1 w}(s)$, the approximation errors of the frequencyweighted reduced models $\widetilde{G}_{1 w}(s)$ and $\widetilde{G}_{2 w}(s)$ are smaller than those of the unity-weighted reducedorder models $\widetilde{G}_{1}(s)$ and $\widetilde{G}_{2}(s)$, respectively, at low frequencies. Moreover, the steady-state errors of the step responses of $\widetilde{G}_{1 w}(s)$ and $\widetilde{G}_{2 w}(s)$ are considerably improved because the gain errors at the frequency $\omega=0$ become smaller owing to the use of the 


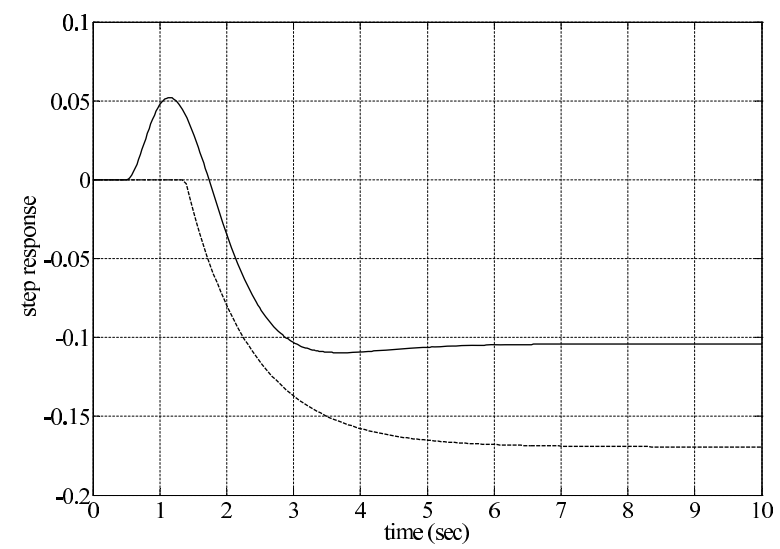

Fig. 9 Step responses of the original system and reduced-order model $\left(-G(s),-\cdots \widetilde{G}_{1}(s)\right)$

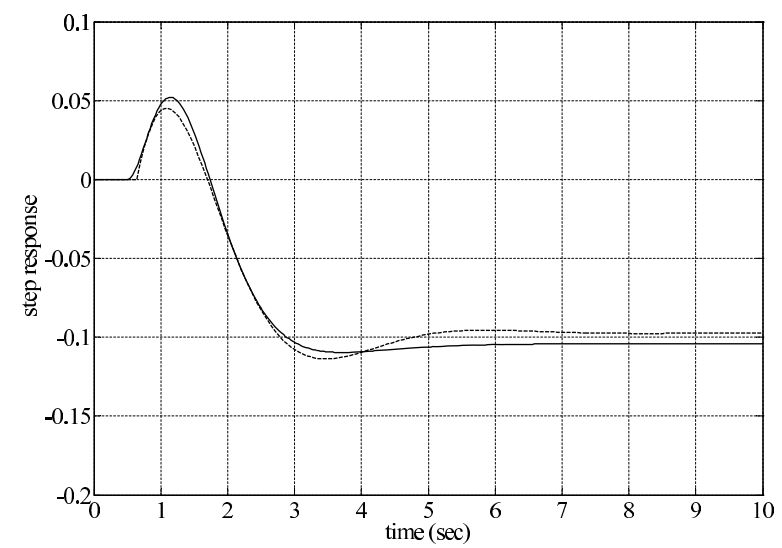

Fig. 10 Step responses of the original system and reduced-order model $\left(-G(s),---\widetilde{G}_{2}(s)\right)$

frequency-weighting function with low-pass characteristics. Therefore, the proposed method can successfully carry out the frequency-weighted model reduction. In control design, the reduction of the steadystate error is one of the important themes. For this purpose, it is effective to choose the frequencyweighting function $W(s)$ so that it has low-pass characteristics.

To carry out a comparison with the GA-based model reduction method [10,11], Monte Carlo simulations with 20 experiments are implemented, where 20 setups of the initial employed bees and the initial population are generated for the $\mathrm{ABC}$ algorithm and the GA. The setting parameters of the GA are chosen as follows:

(i) population size $M=\ell \times 200$

(ii) maximum generation number $g_{\max }=100$

(iii) string length per parameter $r=30$

(vi) crossover probability $P_{c}=0.8$

(v) mutation probability $P_{m}=0.05$
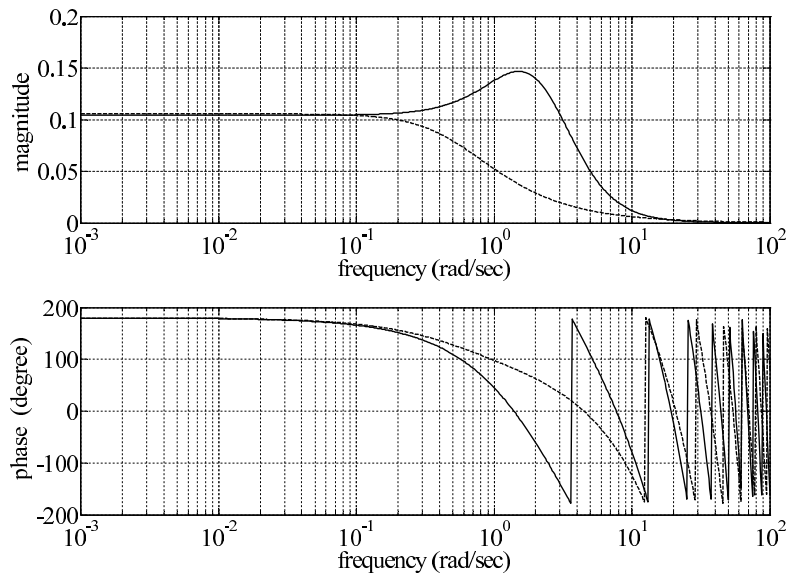

Fig. 11 Bode plots of the original system and frequency-weighted reduced-order model $\quad(-G(s)$, - - $\left.\widetilde{G}_{1 w}(s)\right)$
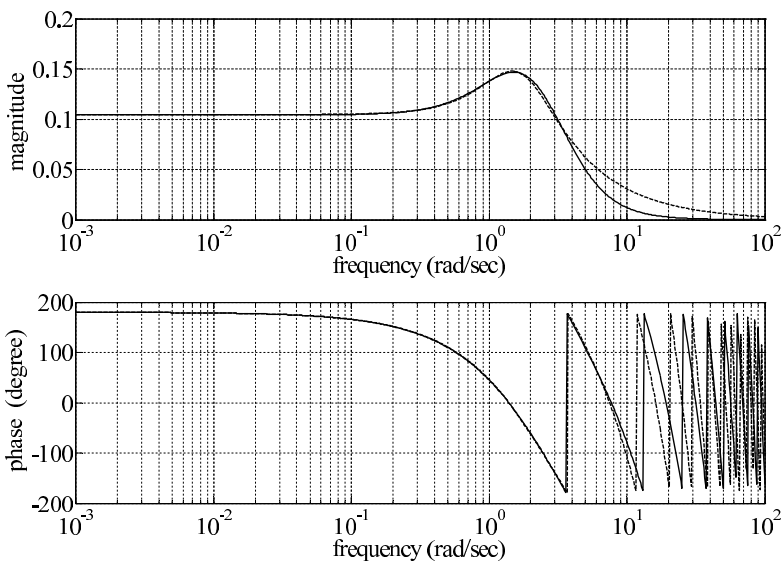

Fig. 12 Bode plots of the original system and frequency-weighted reduced-order model $\quad(-G(s)$, - - $\left.\widetilde{G}_{2 w}(s)\right)$

Note that the number of fitness value calculations of the GA is the same as that of the ABC algorithm. The integrals of the magnitude squared of the transfer function errors in the case of the unity weighting are shown in Table 1 and Fig. 17. Although the transfer function errors of the first-order and second-order reduced models obtained by the proposed method are almost the same as those obtained by the GA-based method, the errors of the third-order and fourth-order reduced models obtained by the proposed method are smaller than those obtained by the GA-based method. Therefore, the proposed method is robust to an increase in the order of the reduced model.

Finally, note that the number of GA parameters is five, whereas the number of ABC parameters is only two. Therefore, the proposed method can yield better reduced-order models with a simpler parameter set- 


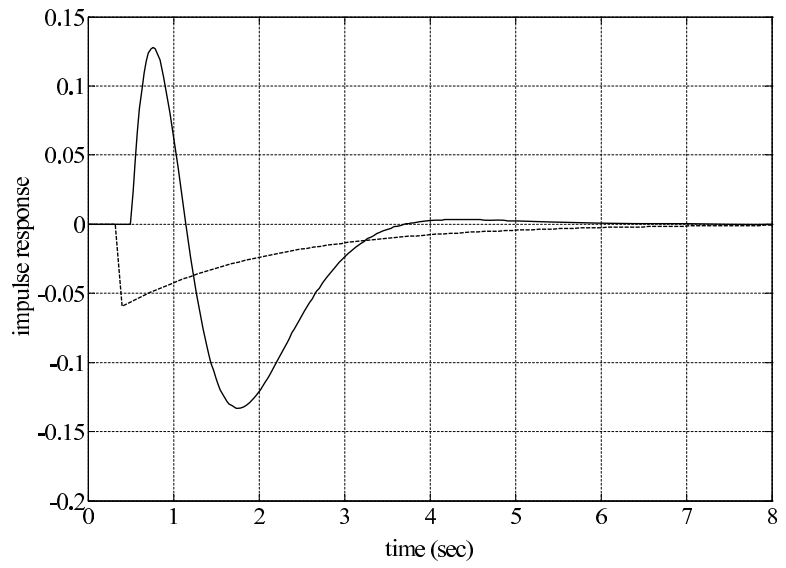

Fig. 13 Impulse responses of the original system and frequency-weighted reduced-order model $(-G(s)$, - - - $\left.\widetilde{G}_{1 w}(s)\right)$

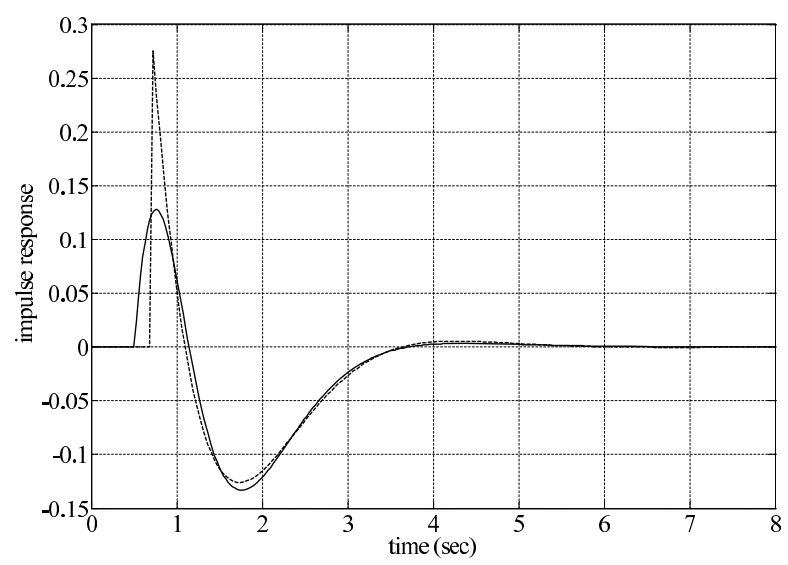

Fig. 14 Impulse responses of the original system and frequency-weighted reduced-order model $(-G(s)$, - - - $\left.\widetilde{G}_{2 w}(s)\right)$

ting than the GA-based method.

\section{Conclusions}

In this paper, a novel method of model reduction with a time delay for single-input, single-output continuous-time systems has been presented using a separable LS approach. The linear LS method and ABC algorithm are efficiently combined to determine the parameters of the rational part and the time delay of the reduced-order model. The denominator parameters and time delay are represented by the positions of the food sources and are searched for by the ABC algorithm, where the candidates of the denominator parameters are estimated by the linear LS method. The use of the ABC algorithm makes the model reduction more accurate and more efficient owing to the

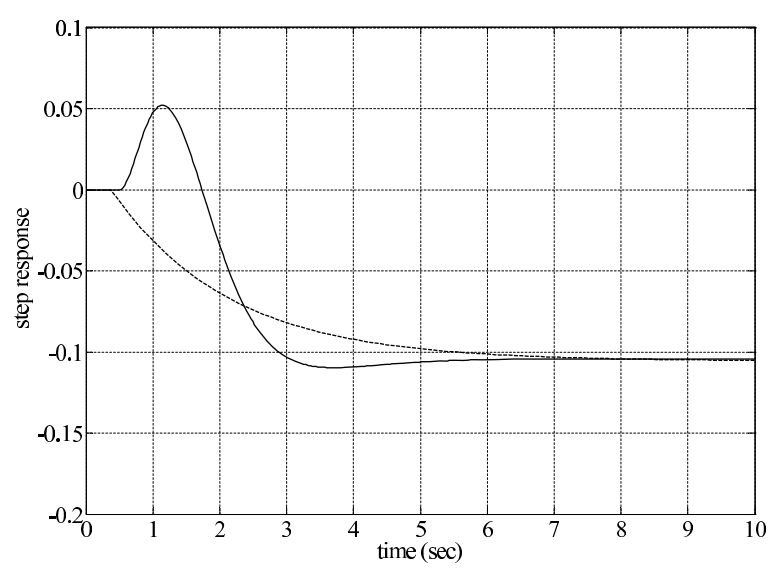

Fig. 15 Step responses of the original system and frequency-weighted reduced-order model ( $-G(s)$, - - - $\left.\widetilde{G}_{1 w}(s)\right)$

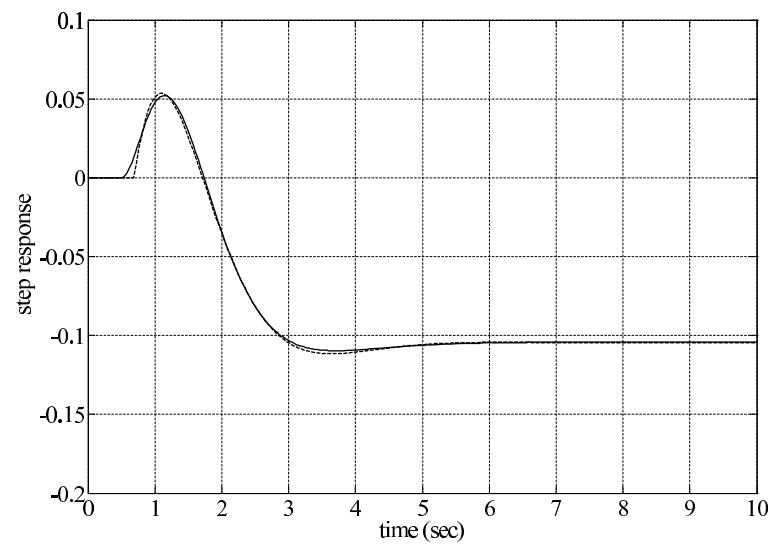

Fig. 16 Step responses of the original system and frequency-weighted reduced-order model ( $G(s)$, - - $\left.\widetilde{G}_{2 w}(s)\right)$

high potential for both global and local optimizations and the simplicity of the ABC algorithm. Simulation results show that the reduced-order models obtained by the proposed method yield good approximations to the original system for both unity-weighted and frequency-weighted model reduction. Simulation results also show that the accuracy of the proposed method is comparable or superior to that of the GAbased model reduction method in spite of fewer setting parameters.

\section{References}

[1] A. Gruca and P. Bertrand: Approximation of high-order systems by low-order models with delays, Int. J. Control, Vol. 28, No. 6, pp. 953-965, 1978. 
Table 1 Integrals of the magnitude squared of the transfer function errors

\begin{tabular}{|c|c|c|}
\hline model & Proposed method & GA-based method \\
\hline$\widetilde{G}_{1}(s)$ & $2.119 \mathrm{e}-2$ & $2.120 \mathrm{e}-2$ \\
$\widetilde{G}_{2}(s)$ & $2.879 \mathrm{e}-3$ & $2.889 \mathrm{e}-3$ \\
$\widetilde{G}_{3}(s)$ & $5.346 \mathrm{e}-5$ & $1.091 \mathrm{e}-4$ \\
$\widetilde{G}_{4}(s)$ & $7.150 \mathrm{e}-6$ & $2.515 \mathrm{e}-5$ \\
\hline
\end{tabular}

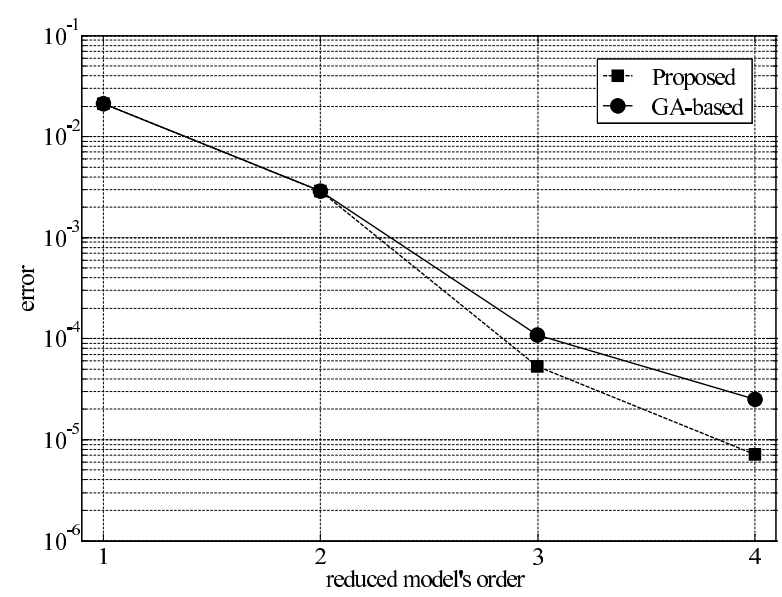

Fig. 17 Integrals of the magnitude squared of the transfer function errors

[2] B. C. Moore: Principal component analysis in linear systems: controllability, observability, and model reduction, IEEE Trans. Autom. Control, Vol. AC-26, No. 1, pp. 1732, 1981.

[3] D. F. Enns: Model reduction with balanced realizations: an error bound and a frequency weighted generalization, Proc. 23rd Conf. on Decision and Control, pp. 127-132, 1984.

[4] K. Glover: All optimal Hankel-norm approximations of linear multivariable systems and their $L^{\infty}$-error bounds, Int. J. Control, Vol. 39, No. 6, pp. 1115-1193, 1984.

[5] Y. Liu and B. D. O. Anderson: Model reduction with time delay, IEE Proc. Pt. D, Vol. 134, No. 6, pp. 349-367, 1987.

[6] W. X. Zheng and C. B. Feng: Hybrid method for model reduction with time delay, Int. J. Syst. Sci., Vol. 21, No. 4 pp. 755-763, 1990.

[7] Y. Halevi: Optimal reduced order models with delay, Proc. 30th Conf. on Decision and Control, pp. 602-607, 1991.

[8] J. T. Spanos, M. H. Milman and D. L. Mingori: A new algorithm for $L_{2}$ optimal model reduction, Automatica, Vol. 28, No. 5, pp. 897-909, 1992.

[9] D. Xue and D. P. Atherton: A suboptimal reduction algorithm for linear systems with a time delay, Int. J. Control, Vol. 60, No. 2, pp. 181-196, 1994.

[10] T. Hachino, Z. J. Yang and T. Tsuji: $L_{2}$ optimal model reduction using the genetic algorithm, Proc. 1st Asian Control Conference, pp. 481-484, 1994.

[11] Z. J. Yang, T. Hachino and T. Tsuji: Model reduction with time delay combining the least-squares method with the genetic algorithm, IEE Proc. Pt. D, Vol. 143, No. 3, pp.
247-254, 1996.

[12] G. H. Golub and V. Pereyra: The differentiation of pseudoinverses and nonlinear least squares problems whose variables separate, SIAM J. Numer. Anal., Vol. 10, No. 2, pp. 413-432, 1973.

[13] J. Bruls, C. T. Chou, B. R. J. Haverkamp and M. Verhaegen: Linear and non-linear system identification using separable least-squares, Eur. J. Control, Vol. 5, pp. 116-128, 1999.

[14] F. Previdi and M. Lovera: Identification of non-linear parametrically varying models using separable least squares, Int. J. Control, Vol. 77, No. 16, pp. 1382-1392, 2004.

[15] T. Hachino, K. Shimoda and H. Takata: Hammerstein system identification by combining least-squares method with particle swarm optimization, J. Signal Process., Vol. 12, No. 6, pp. 497-505, 2008.

[16] D. Karaboga and B. Basturk: On the performance of artificial bee colony (ABC) algorithm, Appl. Soft Comput., Vol. 8, No. 1, pp. 687-697, 2008.

[17] D. Karaboga and B. Akay: A comparative study of artificial bee colony algorithm, Appl. Math. Comput., Vol. 214, No. 1, pp. 108-132, 2009.

[18] I. Iimura and S. Nakayama: Search performance evaluation of artificial bee colony algorithm on high-dimensional function optimization (in Japanese), Trans. ISCIE, Vol. 24, No. 4, pp. 97-99, 2011.

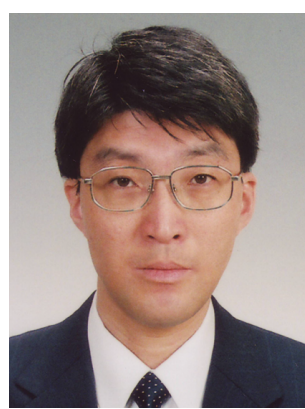

Tomohiro Hachino received his B.S., M.S., and Dr. Eng. degrees in electrical engineering from Kyushu Institute of Technology in 1991, 1993, and 1996, respectively. $\mathrm{He}$ is currently an Associate Professor at the Department of Electrical and Electronics Engineering, Kagoshima University. His research interests include nonlinear control and identification, signal processing, and evolutionary computation. Dr. Hachino is a member of IEEJ, SICE, and ISCIE.

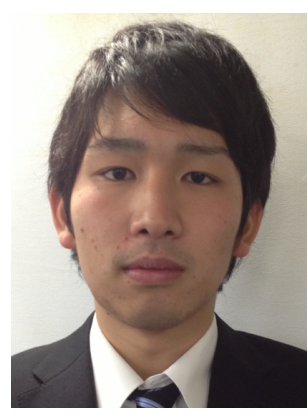

Kosuke Sameshima received his B.S. degree in electrical and electronics engineering from Kagoshima University in 2013. In 2013, he joined West Nippon Expressway Company. His research interests include model reduction and evolutionary computation. 


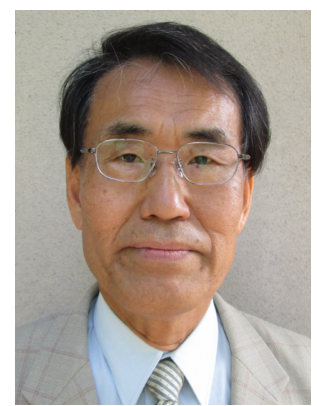

Hitoshi Takata received his B.S. degree in electrical engineering from Kyushu Institute of Technology in 1968 and his M.S. and Dr. Eng. degrees in electrical engineering from Kyushu University in 1970 and 1974, respectively. He is currently a Professor Emeritus and a part-time lecturer at Kagoshima University. His research interests include the control, linearization, and identification of nonlinear systems.

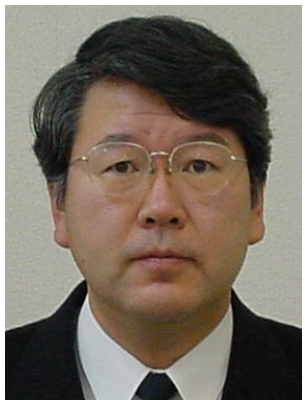

Shigeru Nakayama received his B.S. degree from Kyoto Institute of Technology in 1972, and his M.S. and Dr. Eng. degrees from Kyoto University in 1974 and 1977, respectively. $\mathrm{He}$ is currently a Professor at the Department of Information Science and Biomedical Engineering, Kagoshima University. His research interests include distributed parallel processing, parallel genetic algorithms, parallel image processing, and distributed objects. Dr. Nakayama is a member of IPSJ, IEICE, IEEJ, ISCIE, and JSEE.

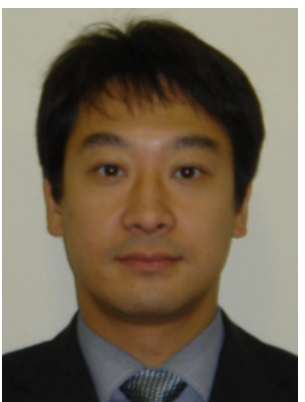

Ichiro Iimura received his B.S. and M.S. degrees from Sophia University in 1992 and 1994, respectively, and his Dr. Eng. degree from Kagoshima University in 2004. He is currently a Professor at the Faculty of Administration, the Prefectural University of Kumamoto. His research interests include evolutionary computation, swarm intelligence, distributed parallel processing, and natural user interfaces. Dr. Iimura is a member of IPSJ,

IEICE, IEEJ, ISCIE, JSAI, and JPNSEC.

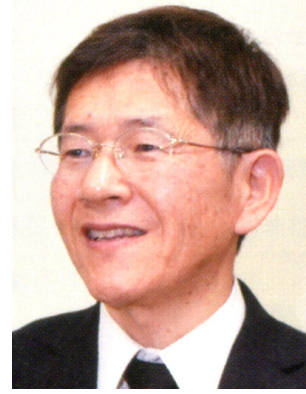

Seiji Fukushima

received his B.S., M.S., and Ph.D, degrees in electrical engineering from Kyushu University in 1984, 1986, and 1993, respectively. $\mathrm{He}$ is currently a Professor at the Department of Electrical and Electronics Engineering, Kagoshima University. His current research interests include photonics/radio hybrid communication systems and their related devices. Dr. Fukushima is a member of IEICE, IEEE/Photonics Society, Japan Society of Applied Physics, Japanese Liquid Crystal Society, and Optical Society of America.

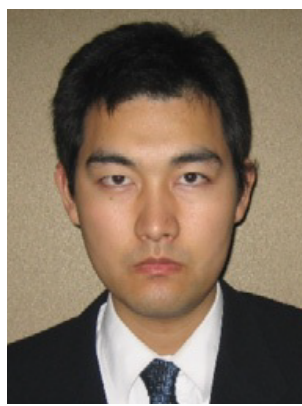

Yasutaka Igarashi received his B.E., M.E., and Ph.D. degrees in information and computer sciences from Saitama University in 2000, 2002, and 2005, respectively. He was a research fellow of Japan Society for the Promotion of Science from 2004 to 2006 . From 2006 to 2011, he was a research associate of Tokyo University of Science. Since 2011, he has been an Assistant Professor of Kagoshima University. $\mathrm{He}$ is involved in research on optical CDMA communication systems and the cryptanalysis of symmetric-key cryptography. Dr. Igarashi is a member of IEICE, and RISP.

(Received March 1, 2013; revised July 9, 2013) 